\title{
Convecção Mantélica com Viscosidade Dependente da Temperatura: Experimentos Numéricos
}

Luiz Gabriel Souza de Oliveira, Natália Valadares de Oliveira - DCN/CEUNES/UFES

Copyright 2014, SBGf - Sociedade Brasileira de Geofísica

Este texto foi preparado para a apresentação no VI Simpósio Brasileiro de Geofísica, Porto Alegre, 14 a 16 de outubro de 2014. Seu conteúdo foi revisado pelo Comitê Técnico do VI SimBGf, mas não necessariamente representa a opinião da SBGf ou de seus associados. É proibida a reprodução total ou parcial deste material para propósitos comerciais sem prévia autorização da SBGf.

\section{Resumo}

Simulações do processo de convecção mantélica terrestre, com viscosidade dependente da temperatura, realizadas com auxílio do código computacional CITCOMS mostraram-se como ferramentas eficientes no estudo da tranferência de calor e massa no interior do planeta, podendo fornecer insights sobre sua relação com os processos geodinâmicos vigentes. A estratificação viscosidade apresentou um comportamento reológico, em termos convectivos, mais realista para o interior do planeta Terra.

\section{Introdução}

O entendimento dos processos geodinâmicos ocorrentes em nosso planeta envolvem, necessariamente, inferências baseadas em informações geológicas, geofísicas e geoquímicas do interior terrestre.

Neste contexto, o processo de convecção mantélica assume um papel chave na atividade tectônica da Terra. O resfriamento convectivo de um planeta rochoso, assumindo a estratificação estável da viscosidade dinâmica de seu manto, que é fortemente dependente da temperatura, é um dos pilares na evolução termal do manto litosférico oceânico, da litosfera cratônica, dos orógenos e das bacias sedimentares. Mantos silicáticos de planetas terrestres deformam-se por meio de fluxo lento (creep), assumindo um comportamento semelhante ao de um fluido de alta viscosidade dinâmica, em longas escalas de tempo geológico (Zaranek \& Parmentier 2004).

Sendo assim, neste trabalho, foram desenvolvidas simulações tridimensionais do processo de convecção mantélica no interior da Terra, assumindo reologia fortemente dependente da temperatura, com soluções numéricas envolvendo o Método dos Elementos Finitos (Hughes 1987).

Os resultados deste tipo de abordagem do problema convectivo no interior terrestre podem fornecer importantes pistas na investigação da evolução geodinâmica do planeta.

\section{Metodologia/ Problema Investigado}

O manto é tratado como um meio viscoso incompressível, onde a convecção termal é controlada pelas equações de conservação de massa, energia e momemento (Chandrasekhar 1961):

$$
\begin{gathered}
\frac{\partial u_{i}}{\partial x_{i}}=0 \\
\frac{-\partial P}{\partial x_{i}}+\left[\eta \frac{\partial u_{i}}{\partial x_{j}}+\eta \frac{\partial u_{j}}{\partial x_{i}}\right]+\delta \rho g \delta_{i r}=0 \\
\frac{\partial T}{\partial t}+u_{i} \frac{\partial T}{\partial x_{i}}=\kappa \frac{\partial^{2} T}{\partial x_{i}^{2}}+H
\end{gathered}
$$

onde $u_{i}$ é a velocidade, $P$ é a pressão dinâmica, $\delta \rho$ a anomalia de densidades, $g$ a aceleração gravitacional, $\eta$ a viscosidade dinâmica, $T$ a temperatura, $\kappa$ a difusividade termal e $H$ a taxa de produção de calor radiogênico. Nesta abordagem, as forças de flutuabilidade associadas à composição e mudanças de fase são ignoradas. Os efeitos do campo gravitacional, da rotação do planeta e do calor radiogênico do manto também são desprezados.

Soluções numéricas das equações (1), na forma adimensionalizada, foram obtidas com o emprego do código computacional envolvendo processamento paralelo CITCOMS (Zhong et al. 2000, Tan et al. 2006, Tan et al. 2012). Trabalhando com malhas esféricas e aplicando o método dos elementos finitos, com base no algoritmo de Uzawa e o método de Petrov-Galerkin, é possível simular o processo convectivo no manto terrestre envolvendo diversos parâmetros e situações geológicas, possibilitando investigar em detalhe 0 processo convectivo e suas consequências geodinâmicas (Tan et al. 2012).

Neste trabalho, assumimos a dependência da viscosidade em função da temperatura por meio da seguinte relação exponencial:

$$
\eta(T)=\eta_{0} \exp \left[\frac{E_{a}+P V_{a}}{R T}\right]
$$

sendo $E_{a}$ a energia de ativação, $V_{a}$ o volume de ativação, $\mathrm{P}$ a pressão hidrostática, $\mathrm{T}$ a temperatura e $\mathrm{R}$ a constante universal dos gases.

Os parâmetros utilizados nas simulações estão apresentados na Tabela 1.

\section{Resultados}

Foram determinadas soluções numéricas para dois casos: um assumindo viscosidade dinâmica constante e outro envolvendo a dependência da mesma em relação à temperatura.

As modelagens desenvovidas neste estudo visaram uma equivalência de tempo de cerca de 400 milhões de anos para a convecção mantélica. Devido ao fato de 
ser um código que envolve processamento paralelo, o CITCOMS resolve os problemas propostos de maneira eficiente, do ponto de vista do tempo computacional.

Como parâmetro adimensional básico que define o início da instabilidade aasociada ao escoamento incompressível convectivo do manto terrestre, levou-se em consideração o número de Rayleigh $R a$ (Chandrasekar 1961):

$$
R a=\frac{\rho g \alpha \Delta T D^{3}}{\eta_{0} \mathrm{~K}}
$$

onde $\Delta T$ é o contraste de temperaturas entre a interface manto-núcleo e a base da litosfera, $D$ a espessura do manto e $\alpha$ o coeficiente de expansão termal.

Tabela 1 - Parâmetros utilizados na modelagem (Schubert et al. 2001)

\begin{tabular}{|c|c|}
\hline Parâmetro & Valores \\
\hline$R a$ & $5 \times 10^{8}$ \\
\hline$\kappa$ & $10^{-6} \mathrm{~m}^{2} / \mathrm{s}$ \\
\hline$\alpha$ & $3 \times 10^{-5} \mathrm{~K}^{-1}$ \\
\hline$\rho$ & $3340 \mathrm{~kg} / \mathrm{m}^{3}$ \\
\hline$g$ & $9.8 \mathrm{~m} / \mathrm{s}^{2}$ \\
\hline$\Delta T$ & $1800 \mathrm{~K}$ \\
\hline$\eta_{0}$ & $10^{21} \mathrm{~Pa} . \mathrm{s}$ \\
\hline$E a$ & $300 \mathrm{~kJ} / \mathrm{mol}^{-6}$ \\
\hline$V a$ & $6 \times 10^{-6} \mathrm{~m} / \mathrm{mol}$ \\
\hline$R$ & $8,31 \mathrm{~J} / \mathrm{K} . \mathrm{mol}$ \\
\hline Raio do planeta & $6371 \mathrm{~km}$ \\
\hline Step computacional & $7.77 \times 10^{-10}$ \\
\hline
\end{tabular}

Foram selecionados seções contendo ao eixo de rotação terrestre, que apresentam as distribuições de temperatura em momentos de tempo específicos. Também foram construídos perfis de temperatura em função da distância radial, na região polar norte da Terra (Figuras 1 e 2).

Os resultados obtidos nos dois experimentos numéricos são sintetizados s seguir:

i) o início do processo convectivo é mais tardio quando a viscosidade é assumida constante (cerca de 160 milhões de anos);

ii) na situação de viscosidade dependente da temperatura, o início da instabilidade ocorre por volta de 40 milhões de anos;

iii)o comprimento de onda da perturbação convectiva é maior no segundo caso;

iv) a velocidade quadrática média $\left(\mathrm{V}_{\mathrm{rms}}\right)$ no caso isoviscoso é menor $\left(5,86 \times 10^{-7} \mathrm{~m} / \mathrm{s}\right)$ do que no caso da viscosidade dependente de $\mathrm{T}\left(7,92 \times 10^{-7} \mathrm{~m} / \mathrm{s}\right)$;

v) a estrutura termal do manto evolui de maneira diferenciada, em ambos os casos;

vi) a homogenização das temperaturas mantélicas ocorre de maneira diferenciada em relação à estrutura de viscosidade dinâmica do manto; vii) é possível notar, no segundo caso, o início da formação do processo de "plumas frias";

viii) o manto tende a homogeneizar sua estrutura de viscosidade com a evolução do processo convectivo, no segundo caso.

\section{Discussão e Conclusões}

Modelos computacionais obtidos por meio de processamento paralelo podem constituir importantes ferramentas de pesquisa na estrutura e evolução da convecção mantélica terrestre.

Neste trabalho foi investigada a influência da variação da viscosidade dinâmica no processo convectivo. Mesmo aplicando modelos simplificados, os resultados alcançados permitiram concluir que o processo convectivo ocorre de maneira mais complexa do que no caso de um manto isoviscoso. Diferentes feições na distribuição de temperaturas e viscosidades no manto podem ser associadas a diversidade de processos geodinâmicos.

Combinando a interpretação dos resultados a dados geofísicos, geológicos e geoquímicos, é possível traçar a evolução termal do manto, e correlacionar o resultado aos processos geodinâmicos atuantes no planeta, que culminam na interação das placas litosféricas, gerando os mais diversos cenários geotectônicos.

Pretende-se, no futuro, investigar o efeito da compressibilidade na convecção mantélica, bem como o da zonação reológico-composicional no manto, dada as presenças da zonas $410 \mathrm{~km}$ e $670 \mathrm{~km}$ da região de transição.

\section{Agradecimentos}

Ao Computational Infrastructure for Geodynamics (CIG) pela disponibilidade do código CITCOMS. Ao Departamento de Ciências Naturais (DCN/CEUNES/UFES) pela infraestrutura computacional.

\section{Referências}

Chandrasekhar, S. 1961. Hydrodynamic and Hydromagnetic Stability. Oxford University Press.

Hughes, T.J.R., 1987. The Finite Element Method: Linear Static and Dynamic Finite Element Analysis. Prentice-Hall.

Schubert, G., Turcotte, D.L. \& Olson, P. 2001. Mantle Convection in Earth and Planets. Cambridge University Press.

Tan, E. et al. (04 co-autores), 2006. Geoframework: Coupling multiple models of mantle convection within a computational framework. Geochemistry, Geophysics, Geosystems, 7: Q06001.

Tan, E. et al. (4 co-autores). 2012. CitcomS - User Manual. Computational Infrastructure for Geodynamics, $132 p$.

Zaranek, S.E. \& Parmentier, E.M., 2004. Convective cooling of an initially stably stratifeied fluid with temperature-dependent viscosity: Implications for the role of solid-state convection in planetary evolution. Journal of Geophysical Research, 109: B033409. 
Zhong, S. et al. (03 co-autores), 2000. Role of temperature-dependent viscosity and surface plate in spherical shell models of mantle convection. Journal of Geophysical Research, 105: 11063-11082.

a)

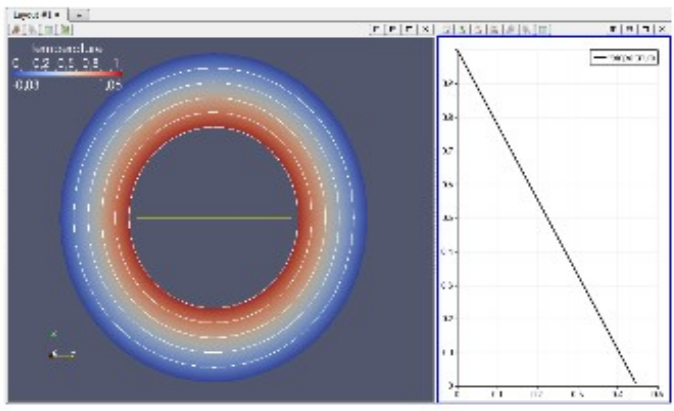

c)

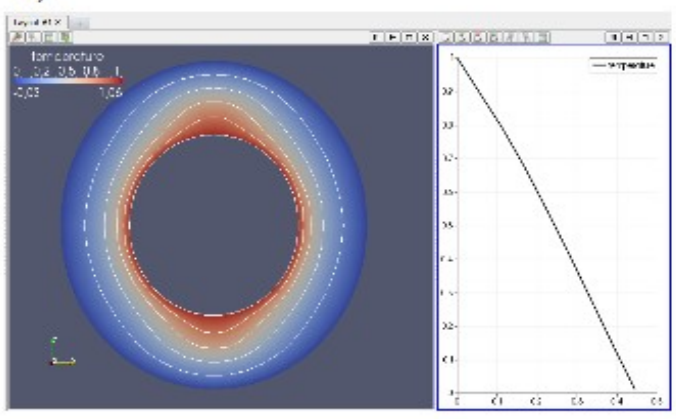

e)

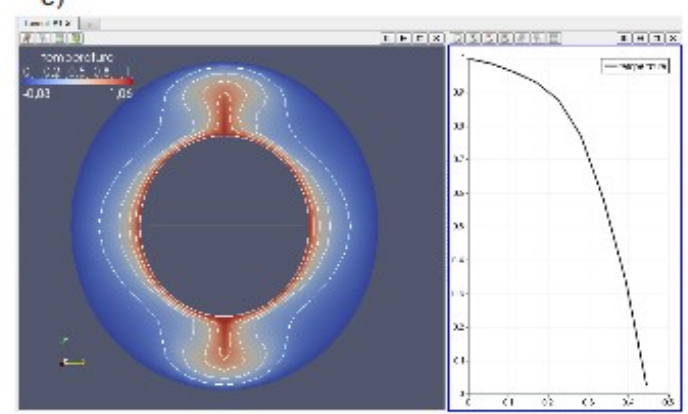

b)

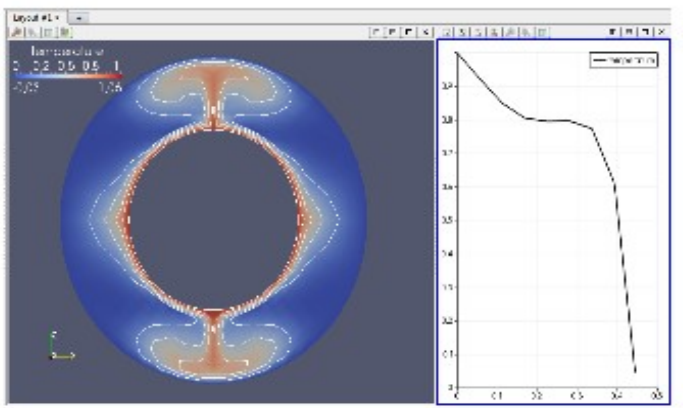

d)

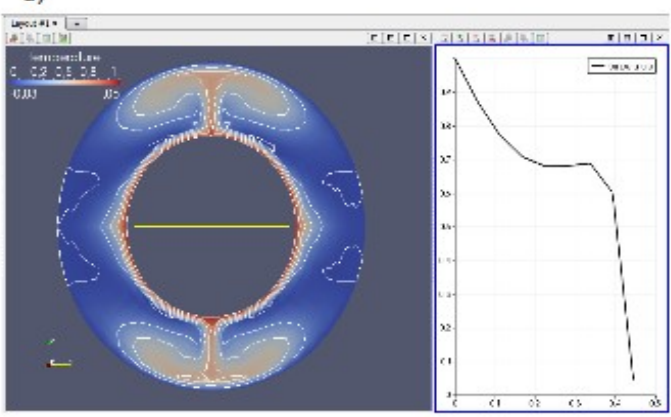

f)

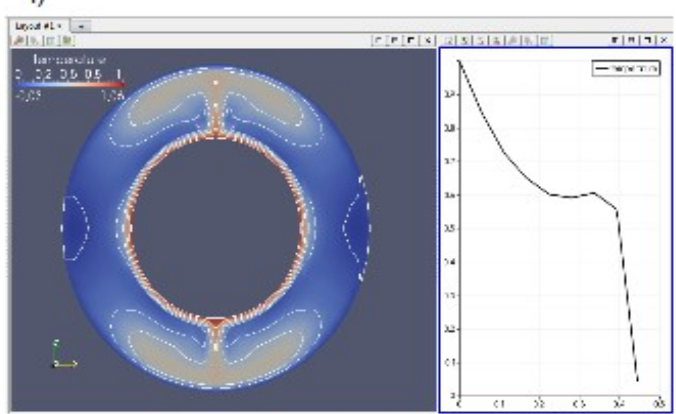

Figura 1 - Seções transversais ao equador terrestre apresentando o processo convectivo do manto, considerado isoviscoso ( $\eta=10^{21} \mathrm{~Pa} . \mathrm{s}$ ) em a) $0 \mathrm{Ma}$; b) $180 \mathrm{Ma}$; c) $230 \mathrm{Ma}$; d) $280 \mathrm{Ma}$; e) $330 \mathrm{Ma}$ e f) $400 \mathrm{Ma}$. Temperaturas normalizadas para cada momento no tempo também são apresentadas. Contornos de temperatura adimensionalizada $=0.2$. 
a)

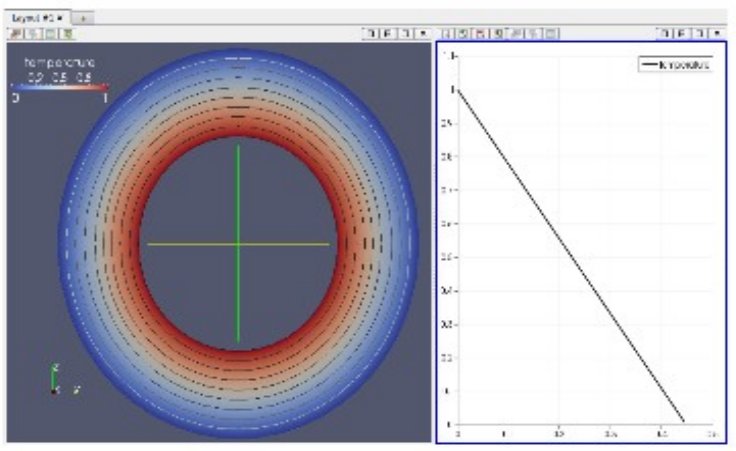

c)

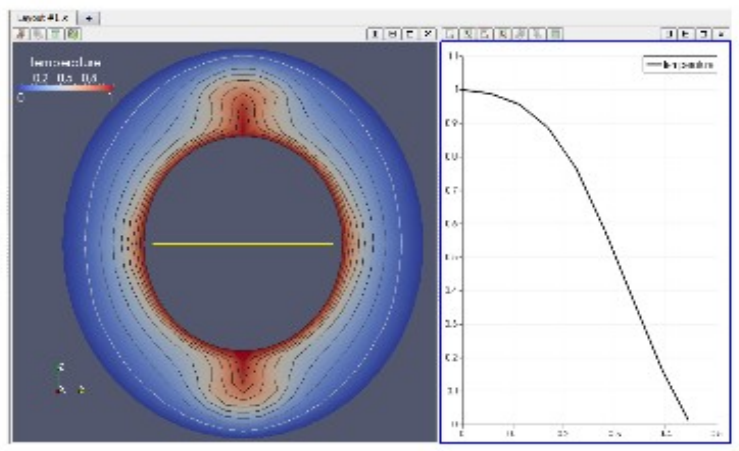

e)

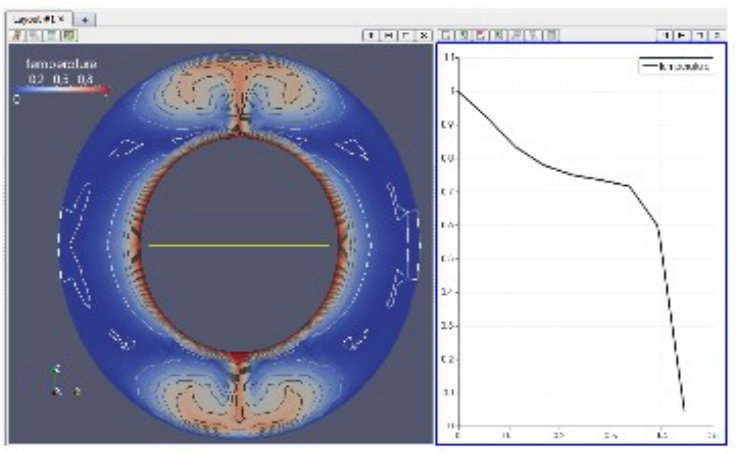

b)

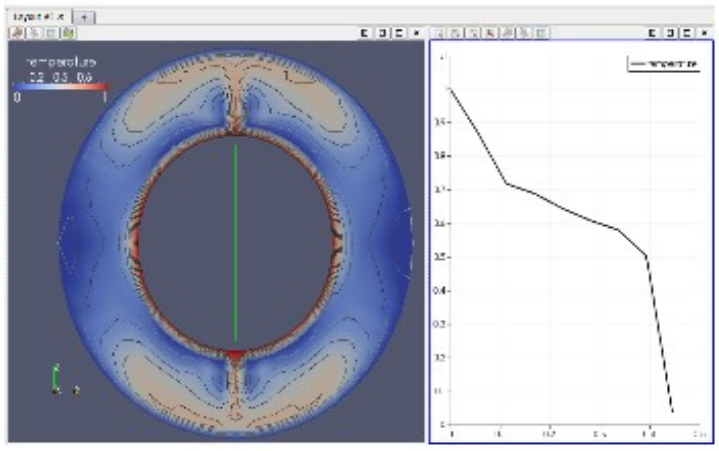

d)

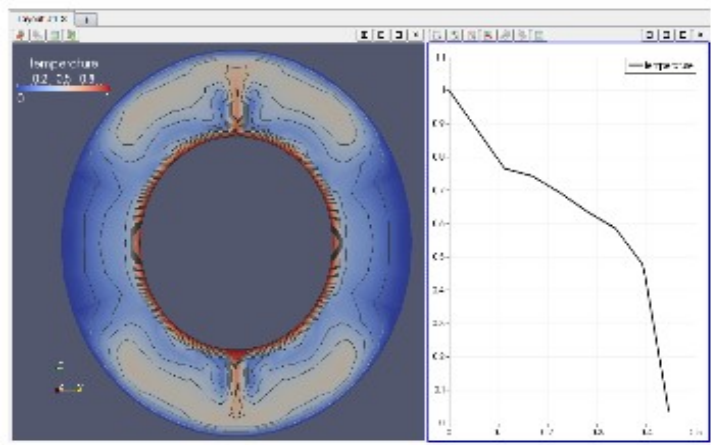

f)

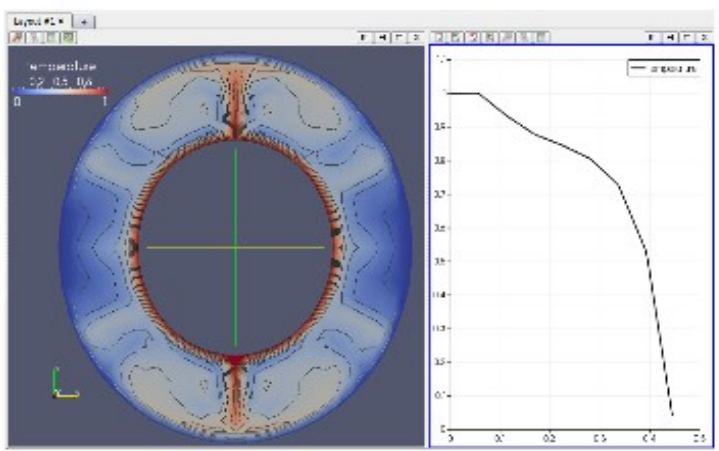

Figura 2 - Seções transversais ao equador terrestre apresentando o processo convectivo do manto, com viscosidade dependente da temperatura, em a) $0 \mathrm{Ma}$; b) $50 \mathrm{Ma}$; c) $80 \mathrm{Ma}$; d) $150 \mathrm{Ma}$; e) $250 \mathrm{Ma}$ e f) $400 \mathrm{Ma}$. Temperaturas normalizadas para cada momento no tempo também são apresentadas. Contornos de viscosidade dinâmica adimensionalizada $=0.2$. 